\title{
A new super-element for estimating the collision resistance of an inclined ship side
}

\author{
L. Buldgen \\ FRIA PhD Student, University of Liège, Liège, Belgium \\ H. Le Sourne \\ Institut Catholique d'Art et Métiers, Nantes, France \\ P.Rigo \\ University of Liège, Liège Belgium
}

\begin{abstract}
This article is directly related to the well-known super-elements technique, used for rapidly estimating the collision resistance of a ship struck by another one. This paper introduces a new type of superelement for considering properly the case of inclined plating. The first part of the article is devoted to a short presentation of the mathematical developments leading to the crushing resistance. The proposed formula is then validated by numerical simulations on individual components. In the second part of the study, we consider the example of a collided ship having inclined plating elements. A comparison is made between the results provided by numerical simulations and those given by a simplified analytical tool involving the abovementioned new super-element.
\end{abstract}

\section{INTRODUCTION}

Amongst all the loads that may act on a ship, it is important to consider the case of a collision between two vessels, especially for ships transporting polluting substances or for military vessels. For checking the resistance to collision, it is of common practice to use finite elements software. As this approach may be time-expensive, some simplified tools were developed for rapidly assessing the ability of a vessel to withstand a collision. Such methods are very useful for optimization in the pre-design phase of the impacted ship.

The basic idea of these simplified procedures is to divide the vessel into structural macro-components, called "super-elements". Each of them is characterized by a relation giving the crushing resistance with respect to the penetration of the striking ship. As the impacting vessel is moving forward into the struck structure, these super-elements are successively activated and their contribution to the total collision force is evaluated.

As a consequence, one of the most important steps is to coherently derive the individual crushing resisting force for the various components. Some results are already available in the literature, especially for horizontal or vertical members. However, the side shell of a vessel may also presents some inclined parts. Some additional research is therefore needed, and it is the aim this paper to present a new super-element for considering properly the case of inclined plating.

\section{ANALYTICAL DEVELOPMENTS}

\subsection{Displacements field}

The super-element under consideration is an inclined plate impacted by a ship bow (Fig. 1). This one is characterized by the parameters $\varphi$ and $\psi$ corresponding respectively to the stem and side angles. The local coordinate frame associated to the vessel is $\left(x_{s}\right.$, $\left.y_{s}, z_{s}\right)$. In this frame, the curve $\Gamma$ describing the shape of the uppermost deck has the following mathematical equation:

$\Gamma \equiv z_{s}=R_{Z}-R_{Z} \frac{x_{s}^{2}}{R_{X}^{2}}$

where $R_{X}$ and $R_{Z}$ are the two radii of the parabola (Fig. 1). The angle between the plate and the uppermost deck is denoted by $\alpha$.

The plate is assumed to be simply supported on its four edges. The initial contact point $\mathrm{P}$ defines four regions characterized by the dimensions $a_{1}, a_{2}, b_{1}$ and $b_{2}$. Local axes $(x, y, z)$ are also attached to the inclined plane and are obtained by rotating the absolute frame $(X, Y, Z)$.

As the ship is moving forwards, the plate is submitted to strong tensile forces implying internal dissipation and it is our purpose to estimate the corresponding energy rate. To do so, it is necessary to postulate the displacements field occurring on the structure during the impact. For a given penetration $\delta$ parallel to the global $Z$ axis, we assume that the plate is only submitted to a displacement $w(x, y)$ acting 
perpendicularly to its plane, i.e. along the local $z$ axis.

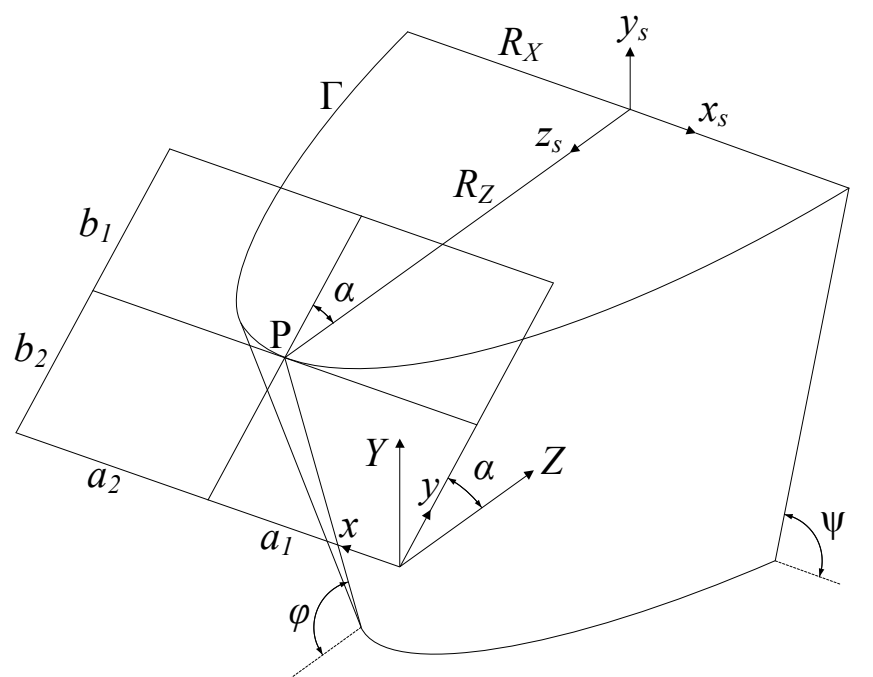

Figure 1. Three dimensional view of the impact scenario.

Let us first consider the plane of the uppermost deck. For a given indentation $\delta$, the plate is forced to follow the shape of the impacting bow, which leads to the deformation depicted on Figure 2. In order to avoid any redundancy, we will only describe the displacements field for the region $0 \leq x \leq a_{1}$, but developments are similar for $a_{1} \leq x \leq a_{1}+a_{2}$.

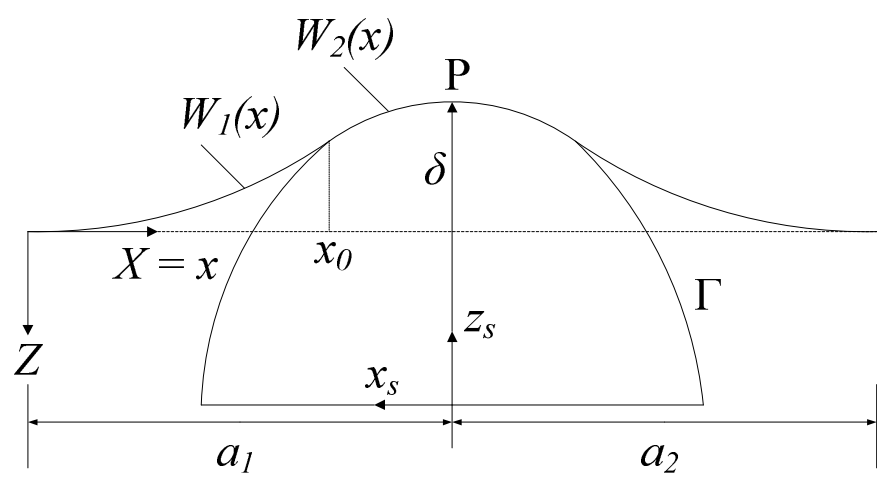

Figure 2. Displacement $W(x)$ in the plane of the uppermost deck.

The displacement field $W(x)$ in the plane of the uppermost deck is composed of two parts. The first part $W_{l}(x)$ is limited to the zone $0 \leq x \leq x_{0}$ and is defined so as to be tangent to the curve $\Gamma$ for $x=x_{0}$. We also assume a horizontal tangency for $x=0$. Considering equation (1) leads to:

$W_{1}(x)=\delta \frac{x^{2}}{a_{1} x_{0}}$

The second part $W_{2}(x)$ has to perfectly fit the curve $\Gamma$, because the plate is supposed to take the same shape as the impacting bow. Consequently, $W_{2}(x)$ may be written as:

$W_{2}(x)=\delta-R_{Z} \frac{\left(x-a_{1}\right)^{2}}{R_{X}^{2}}$
The two previous components $W_{l}(x)$ and $W_{2}(x)$ are separated by the abscissa $x=x_{0}$. Initially, for $\delta=0$, we have $x_{0}=a_{1}$ but for further indentation, $x_{0}$ tends to 0 . If we account for the tangency conditions explained here over, it can be shown that $x_{0}$ is given by:

$x_{0}(\delta)=a_{1}-\frac{R_{X}^{2}}{R_{Z}} \frac{\delta}{a_{1}}$

The displacement $W(x)$ is now entirely characterized by equations (2) to (4). It is worth noting that $W(x)$ is strictly horizontal, i.e. parallel to the global $Z$ axis. However, we postulate that each point $(x, y)$ is only submitted to a movement perpendicular to the plate. To illustrate this assumption, the displacements profile in a plane perpendicular to the $X$ axis is depicted on Figure 3. It can be seen that the shape function $W(x)$ is simply linearly interpolated so as to have $w(x, y)=0$ for $y=0$ and $y=b_{1}+b_{2}$. If we consider the plane perpendicular to the $X$ axis and passing through point $P$ (left part of Figure 3), we see that $\mathrm{P}$ is submitted to the horizontal indentation $W\left(a_{1}\right)=\delta$, but the points of the plate only suffer a displacement $\delta$ sin $\alpha$ decreasing linearly with $y$. Similarly, in any plane perpendicular to the $X$ axis, the maximal displacement is simply given by $w(x, y)=$ $W(x) \sin \alpha$ and is linearly interpolated along the $y$ axis. This can be written as:

- If $0 \leq y \leq b_{2}-W(x) \cos \alpha$, then:

$w(x, y)=\frac{W(x) \sin \alpha}{b_{2}-W(x) \cos \alpha} y$

- If $b_{2}-W(x) \cos \alpha \leq y \leq b_{1}+b_{2}$, then:

$w(x, y)=\frac{W(x) \sin \alpha}{b_{1}+W(x) \cos \alpha}\left(b_{1}+b_{2}-y\right)$

where $W(x)$ is given by equations (2) or (3) according to the position along the $x$ axis.

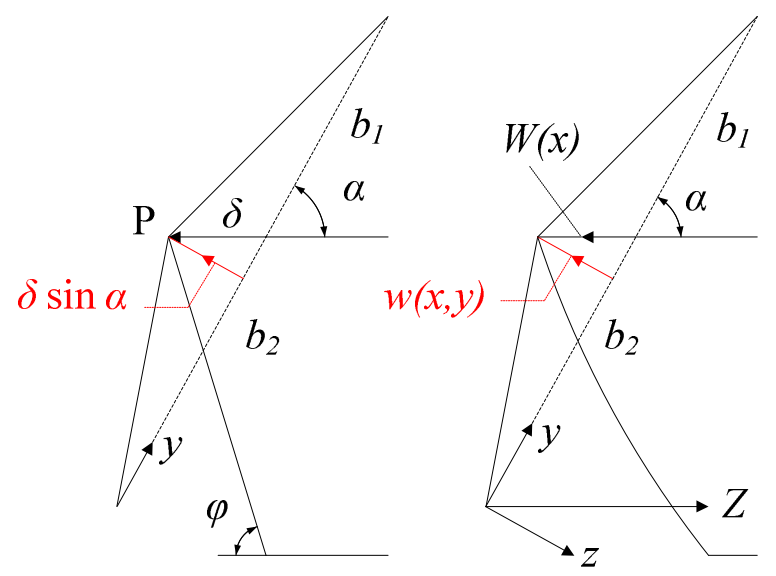

Figure 3. Displacements in a plane parallel to $(Y, Z)$ plane.

This assumption of having $w(x, y)$ perpendicular to the plate means that the structure is submitted to a 
plastic flow, for which each material point is simply "flowing" over the bow. Of course, this implies that no friction is likely to take place between the stem and the structure, which seems rather conservative as the effective penetration $\delta$ tends thus to be overestimated.

\subsection{Internal energy rate}

Equations (5) and (6) describe the displacements function $w(x, y)$ for every point $(x, y)$ belonging to the surface of the plate. This function is said to be kinematically admissible, as it is compatible and respects all the boundary conditions. For this reason, $w(x, y)$ may be used for applying the upper-bound principle. According to Jones (1997), this theorem states that if the work rate of a system of applied loads during any kinematically admissible collapse of a structure is equated to the corresponding internal energy dissipation rate, then that system of loads will cause (incipient) collapse of the structure. If we denote by $\partial E_{\text {int }}$ the internal energy rate produced by the velocity field $\partial w(x, y)$, the resistance $P$ opposed by the superelement during the collision may be found by the virtual work principle:

$\partial E_{\mathrm{int}}=P \cdot \partial \delta \Leftrightarrow P=\frac{\partial E_{\mathrm{int}}}{\partial \delta}$

According to the above-mentioned upper-bound theorem, it is clear that equation (7) may give an excessive approximation of the real plate resistance if the collapse displacements field $w(x, y)$ is not carefully chosen. Under the hypothesis of a plane stress state, Simonsen (1997) shows that the plastic flow theory leads to the following expression of the internal energy rate:

$$
\dot{E}_{\mathrm{int}}=\frac{2 \sigma_{0} t_{p}}{\sqrt{3}} \iint_{A} \sqrt{\dot{\varepsilon}_{x x}^{2}+\dot{\varepsilon}_{y y}^{2}+\dot{\varepsilon}_{x y}^{2}+\dot{\varepsilon}_{x x} \dot{\varepsilon}_{y y}} d x d y
$$

where the dot is used for denoting the derivative $\partial / \partial \delta, A$ is the area of the plate, $t_{p}$ is the thickness and $\sigma_{0}$ is the flow stress. In this last expression, $\partial \varepsilon_{x x}, \partial \varepsilon_{y y}$ and $\partial \varepsilon_{x y}$ are the components of the strain rate tensor. They may be found by taking the incremental form of the Green-Lagrange relations:

$$
\varepsilon_{x x}=\frac{1}{2}\left(\frac{\partial w}{\partial x}\right)^{2} \quad \varepsilon_{y y}=\frac{1}{2}\left(\frac{\partial w}{\partial y}\right)^{2} \quad \varepsilon_{x y}=\frac{1}{2} \frac{\partial w}{\partial x} \frac{\partial w}{\partial y}
$$

So the internal energy rate $\partial E_{\text {int }}$ may be found by introducing equations (5) and (6) into (9), and then deriving the obtained expressions with respect to $\delta$. This leads to the strain rates associated with the field $w(x, y) . \partial \varepsilon_{x x}, \partial \varepsilon_{y y}$ and $\partial \varepsilon_{x y}$ are then introduced in equation (8) in order to obtain the corresponding energy rate. However, the mathematical formulations of $w(x, y)$ are too complex for allowing an analytical integration of equation (8). As a numerical approach is not directly sought, we will rather make another approximation to get a closed-form solution of $\partial E_{\text {int }}$. In fact, the plate is supposed to be made of independent fibers oriented along the $x$ and $y$ axes. During the collision, these fibers are only submitted to membrane deformations along $x$ or $y$, without suffering any shearing force. Consequently, $\varepsilon_{x y}=0$ and the strain energy associated to $\varepsilon_{x x}$ and $\varepsilon_{y y}$ may be evaluated separately:

$$
\dot{E}_{\mathrm{int}}=\sigma_{0} t_{p} \iint_{A} \dot{\varepsilon}_{x x} d x d y+\sigma_{0} t p \iint_{A} \dot{\varepsilon}_{y y} d x d y
$$

Introducing (9) into the previous equation and taking account of (7) leads to the following expression of the resistance opposed by the plate:

$$
P=\sigma_{0} t \int_{p} \iint_{A}\left(\frac{\partial w}{\partial x} \frac{\partial^{2} w}{\partial x \partial \delta}+\frac{\partial w}{\partial y} \frac{\partial^{2} w}{\partial y \partial \delta}\right) d x d y
$$

where $w(x, y)$ has been defined previously by (5) or (6). Equation (11) may be analytically solved in order to derive a closed-form expression of $P$. However, the developments are quite fastidious and will not be detailed in this paper. So far, it is important to bear in mind that the total resisting force $P$ is the sum of two contributions $P_{x}$ and $P_{y}$ given by:

$$
\begin{aligned}
& P_{x}=\sigma_{0} t_{p} \iint_{A} \frac{\partial w}{\partial x} \frac{\partial^{2} w}{\partial x \partial \delta} d x d y \\
& P_{y}=\sigma_{0} t_{p} \iint_{A} \frac{\partial w}{\partial y} \frac{\partial^{2} w}{\partial y \partial \delta} d x d y
\end{aligned}
$$

where $P_{x}$ and $P_{y}$ are due to the membrane elongation of the fibers oriented along $x$ or $y$ respectively (the detailed mathematical expressions of $P_{x}$ and $P_{y}$ are provided as an appendix). The assumption of a nonshearing displacements field is a conservative approach because it neglects the energy that is also dissipated through the component $\varepsilon_{x y}$ of the strain tensor.

\subsection{Failure}

All the previous developments are valid provided that there is no failure in the material. However, as the indentation is growing, the plate is submitted to increased deformations that finally result in a material rupture. The membrane energy is then released, which in turn causes an abrupt decrease of the resistance. However, after this sudden transition, the resistance tends to stabilize at a non-zero level (Fig. 4). This is due to the fact that tearing is first located in a confined area of the plate, before extending to a larger region. Of course, as tearing develops, the resistance is becoming smaller. In our simplified approach, we will simply admit that the resistance is set to zero immediately after the initiation of tearing (Fig. 4). 


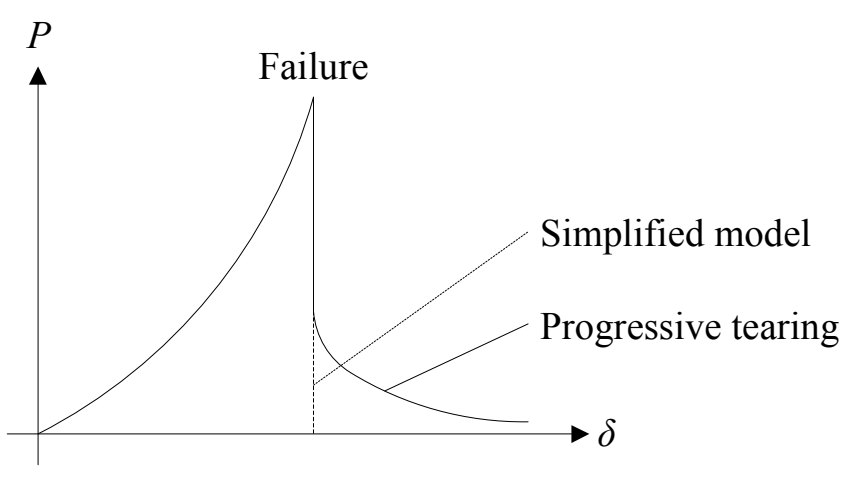

Figure 4. Resistance before/after rupture and present simplified model.

In order to account for this phenomenon, we assume that the fibers oriented along the $x$ (resp. $y$ ) axis are no more able to support tensile forces if their deformations $\varepsilon_{x x}$ (resp. $\varepsilon_{y y}$ ) becomes larger than a given threshold value $\varepsilon_{c}$. This may be written as:

$$
\begin{aligned}
& \varepsilon_{x x}=\frac{1}{2}\left(\frac{\partial w}{\partial x}\right)^{2}>\varepsilon_{c} \rightarrow P_{x}=0 \\
& \varepsilon_{y y}=\frac{1}{2}\left(\frac{\partial w}{\partial y}\right)^{2}>\varepsilon_{c} \rightarrow P_{y}=0
\end{aligned}
$$

where $\varepsilon_{c}$ is the threshold value of the deformation for which rupture is assumed to take place on the superelement. According to Zhang (1999) or Lützen (2001), typical values for $\varepsilon_{c}$ are between 0.06 and 0.12 .

Equations (14) and (15) together with equation (11) completely define the resistance $P$ offered by the plate for a given penetration $\delta$ of the striking ship. The law $P(\delta)$ is precisely the relation sought for characterizing the present new super-element. Because of all the previous hypotheses, the method for deriving $P$ may appear to be over-conservative, but one has to bear in mind that the application of the upper-bound method always provides an excessive approximation for the real resistance of a collapsing structure.

\section{INDIVIDUAL VALIDATION}

In order to validate the expression of $P(\delta)$ for the new inclined super-element, some comparison are made with numerical solutions given by the finite elements code LS-DYNA. To do so, the bow of the striking ship is modeled with rigid shell-elements. Its geometrical properties (as defined on Figure 1) are listed in Table 1.

This ship bow is used for impacting different plates having various lengths $\left(a_{1}+a_{2}\right)$ and $\left(b_{1}+b_{2}\right)$. For each of them, the inclination varies from $\alpha=45^{\circ}$ to $\alpha=90^{\circ}$ with a step of $15^{\circ}$. So intermediate simulations are also performed for $60^{\circ}$ and $90^{\circ}$.
Table 1. Geometrical dimensions of the striking bow as defined on Figure 1.

\begin{tabular}{cccc}
\hline$p(\mathrm{~m})$ & $q(\mathrm{~m})$ & $\varphi(\mathrm{deg})$ & $\psi(\mathrm{deg})$ \\
\hline 6 & 8 & 84 & 84 \\
\hline
\end{tabular}

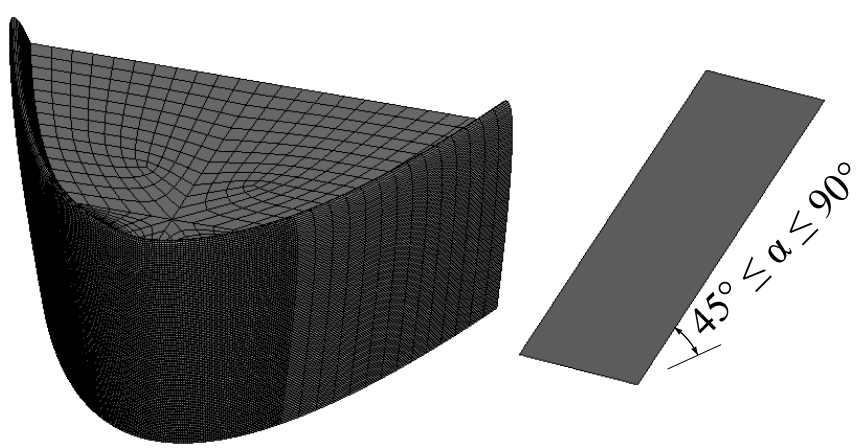

Figure 5. Finite elements models of the striking ship and the struck plate.

The mesh in the contact area is similar for the plate and for the ship, with an element size of $5 \times 5$ $\mathrm{cm}$. The plate is simply supported on its four edges and is collided by the vessel travelling at the initial speed of $2 \mathrm{~m} / \mathrm{s}$. In this paper, we only present results obtained for the following characteristics: $a_{1}=2.5$ $\mathrm{m}, a_{2}=2.5 \mathrm{~m}, b_{1}=2 \mathrm{~m}, b_{2}=2 \mathrm{~m}$ and $t_{p}=0.02 \mathrm{~m}$.

The material law used for the plate is depicted on Figure 6. The stress-strain curve is made of two parts. The first one corresponds to the elastic phase, where a linear behavior is assumed. The stresses are directly proportional to the strain through the Young's modulus $E$, until the elastic capacity $\sigma_{0}$ is reached. The second phase stands for a plastic behavior, with a linear hardening characterized by the tangent modulus $E_{T}$. If an element is deformed beyond the ultimate strain $\varepsilon_{u}$, then rupture occurs and the element is simply removed from the numerical model. For our simulations with LS-DYNA, the parameters listed in Table 2 are used. They are assumed to fit reasonably with classical steel.

Table 2. Parameters defining the stress-strain curve for classical steel, as depicted on Figure 6.

\begin{tabular}{cccc}
\hline$E(\mathrm{MPa})$ & $E_{T}(\mathrm{MPa})$ & $\sigma_{0}(\mathrm{MPa})$ & $\varepsilon_{u}$ \\
\hline 210000 & 1015 & 240 & 0.2 \\
\hline
\end{tabular}

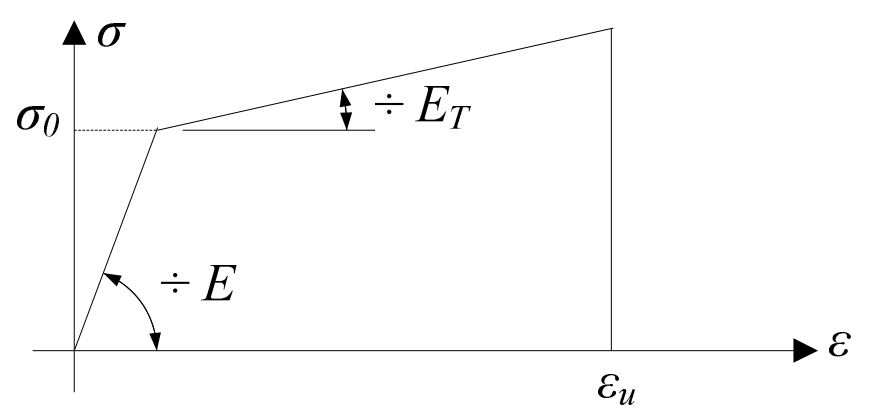

Figure 6. Material law used for numerical simulations. 


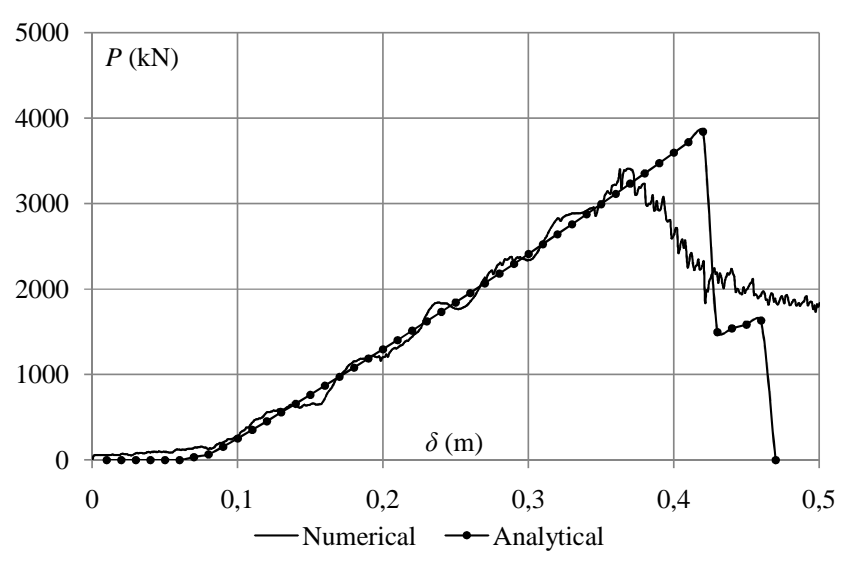

Figure 7. Comparison between analytical and FEM, $\alpha=45^{\circ}$.

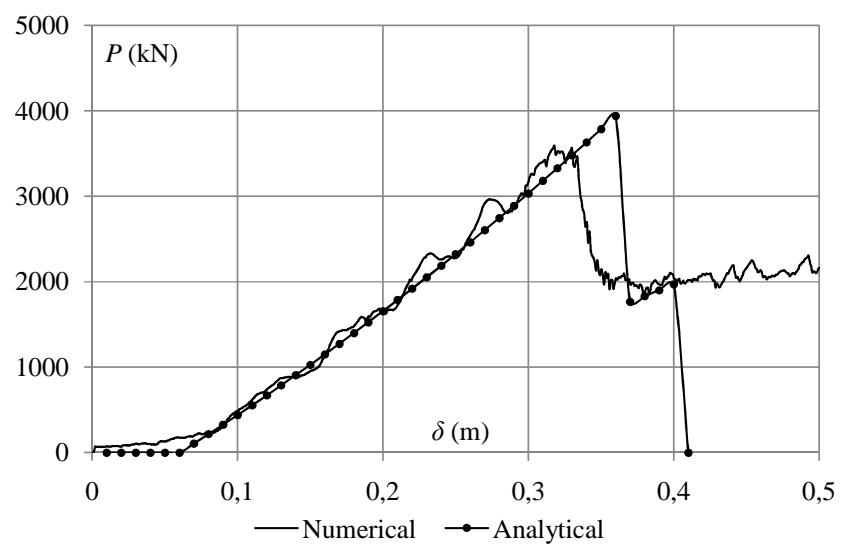

Figure 8. Comparison between analytical and FEM, $\alpha=60^{\circ}$.

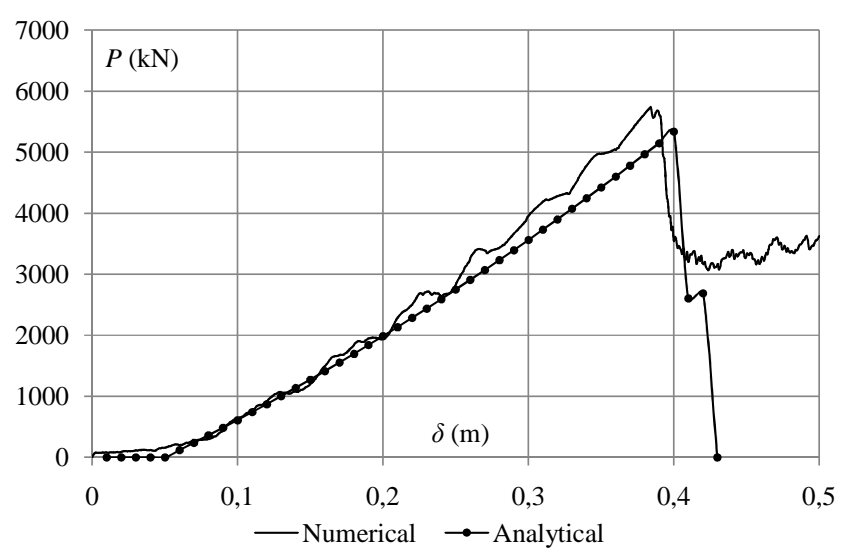

Figure 9. Comparison between analytical and FEM, $\alpha=75^{\circ}$.

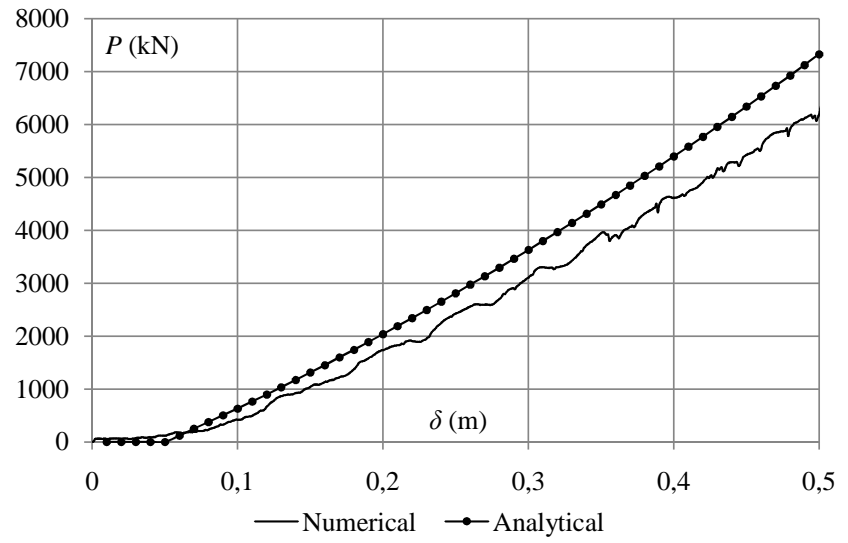

Figure 10. Comparison between analytical and FEM, $\alpha=90^{\circ}$.

Figures 7 to 10 show comparisons between results obtained numerically and the present inclined super-element for the dimensions listed here over. As it can be seen, the resistance is increasing with the angle $\alpha$, which means that vertical panels $(\alpha=$ $90^{\circ}$ ) oppose the largest force to the indentation of the striking vessel.

These figures show that the agreement between the curves is quite good. In some cases however, the analytical failure appears for greater penetrations than in the numerical model. This is mainly due to the difficulty of choosing an appropriate value for the critical strain $\varepsilon_{c}$. In the present paper, we choose $\varepsilon_{c}=8 \%$, as recommended by Zhang (1999) and Lützen (2001) or Buldgen (2012) who mentioned that typical values of $\varepsilon_{c}$ are between 6 and $12 \%$. In fact, we practically determined $\varepsilon_{c}$ by making comparisons with the rupture levels predicted by finite elements analyses, so that the rupture strain predicted by the super-elements method corresponds to the one given by LS-DYNA. For the present inclined super-element, we observed that the most adequate value was $\varepsilon_{c}=8 \%$ because this choice was fitting with most of the numerical results. However, it remains difficult to choose a proper value for $\varepsilon_{c}$ that satisfactorily fit with all numerical simulations.

In the numerical model, rupture is supposed to appear when deformations overcome the value of $\varepsilon_{u}$ $=0.2$, but they are highly dependent on the contact conditions between the stem and the plate. For example, if the striking bow is modeled with too sharp edges, this may results in bad contact conditions involving rupture since the very beginning of the impact. Moreover, as shown by Le Sourne (2001), the numerical failure is also related to the mesh size. For these reasons, we decided not to accord a too large confidence to the precise value of $\delta$ associated with rupture. In addition to that, the discrepancy between numerical and analytical failures remains acceptable.

As mentioned earlier, after failure, the resistance is not immediately set to zero. The plate still withstands further indentations because of the progressive extension of tearing. Since this phenomenon is neglected in the present super-element, our approach remains conservative as it tends to underestimate the total energy dissipated by the structure. This is also an additional argument for not caring about rupture occurring too late.

\section{IMPACT ON A FULL SCALE SHIP}

In this section, the case of a ship collided by the rigid bow depicted on Figure 5 is considered. This scenario will be treated by using both the finite elements code LS-DYNA and the super-elements method. The latter has already been successfully applied by (amongst others) Pedersen \& Zhang (1998), Zhang (1999), Lützen, Simonsen \& Pedersen (2000), Lützen (2001) and Le Sourne (2007) for treating ship-ship collisions. Currently, the applications are 
limited to vessels having an internal structure involving only horizontal and vertical components. However, for some vessels like frigates or aircraft carriers, the shell plating may exhibit a non negligible inclination. So far, this was not tackled by using the super-element approach and the presented new developments are therefore a first step for extending the method.

For the numerical simulations with LS-DYNA, the ship is only represented by one of its sections limited by two transverse bulkheads. The total length is of $11 \mathrm{~m}$ and the first impact point is exactly located at mid-section. Moreover, we suppose that the ship is at rest against a quay, so that no sway motion may occur during the collision. The material characterizing the ship is still the same than the one depicted on Figure 6, with the values listed in Table 2.

In order to point out the importance of accounting for the inclination of the shell plating, we consider two different ships.

\subsection{Ship with slight side inclination}

For this first collision scenario, the shell plating of the ship is not too deeply inclined. A three dimensional view of the internal structure of the vessel is proposed on Figure 11. In order to model the presence of the quay, we apply boundary conditions so as to prohibit any point of the plane $X=0$ from moving in the $Z$ direction. For this first model, the inclination of the shell plating $\alpha=80^{\circ}$ is quite modest. The first contact point between the rigid striking bow and the struck vessel is denoted by $\mathrm{P}$ and depicted on Figure 12.

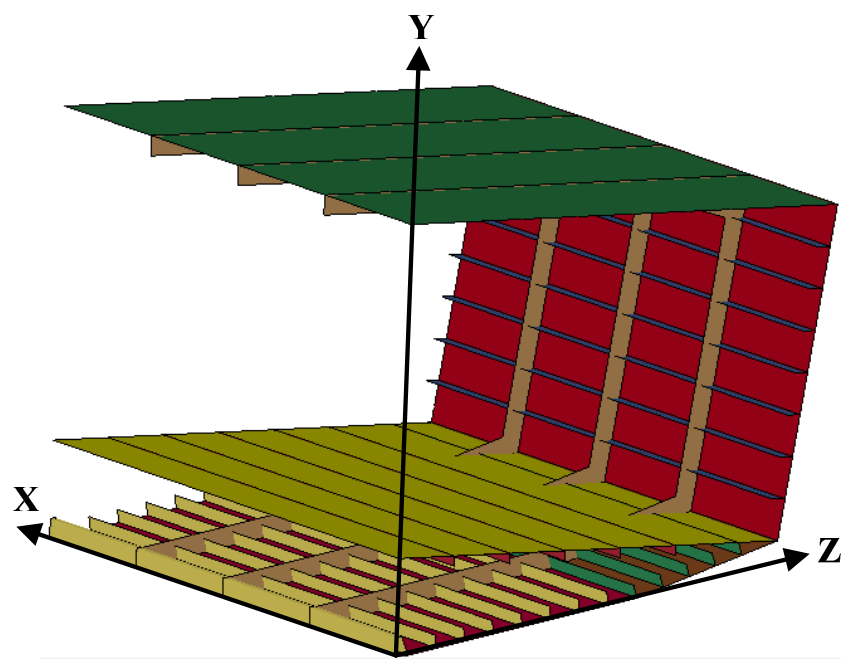

Figure 11. Three dimensional view of ship model 1.

The new inclined super-element developed in this paper has been integrated in a calculation code that allows the ship to be treated as a set of superelements. By so doing, it is possible to derive analytically a rough estimation of the curve showing the evolution of the force $P$ opposed by the ship to the penetration of the rigid bow.

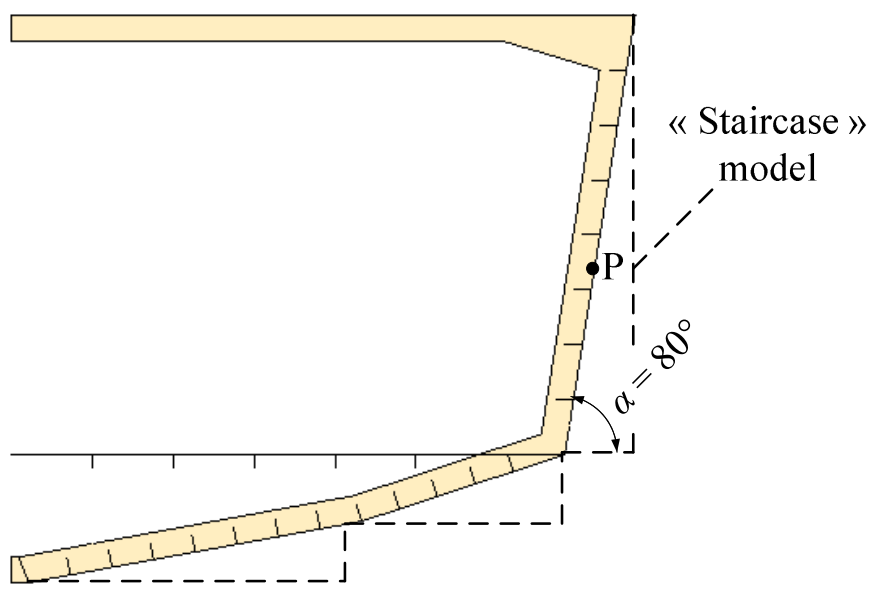

Figure 12. Section of ship model 1 and corresponding "staircase" model.

On Figure 13, we first propose a comparison between the curve $P(\delta)$ given by LS-DYNA ("numerical" curve), and the one obtained by the superelements method including the new developments presented in this paper ("analytical" curve). The agreement is seen to be reasonably good, even if the resistance tends to be overestimated at the beginning of the impact. This is mainly due to a coupling occurring between the uppermost deck (Fig. 11) and the inclined plating. In fact, as the ship is moving forward, this deck is bent around the $X$ axis (Fig. 11) because of the tensile forces transmitted at the boundary with the plating. However, the inclined superelement has been developed under the assumption of a plate simply supported on its four edges. Since the deck exhibits flexural vertical displacements, this hypothesis is not completely realistic and we think that further investigations are still needed in order to account for this phenomenon.

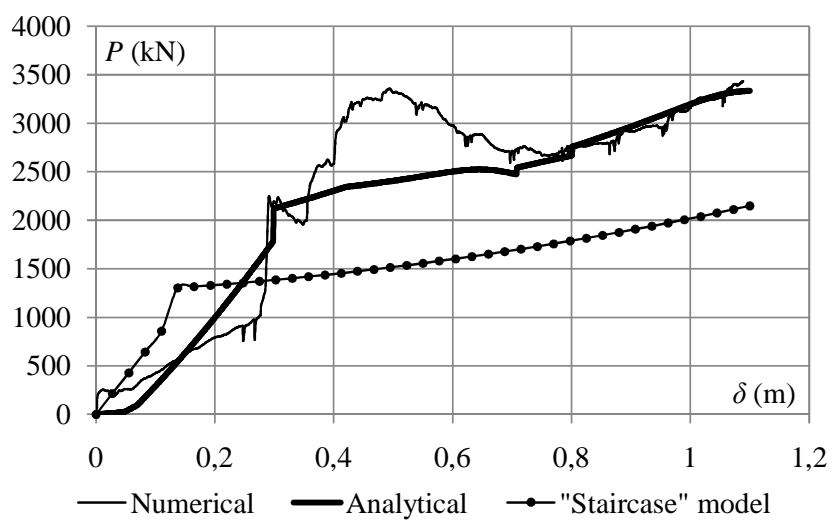

Figure 13. Comparison between numerical and analytical results for ship model 1 .

In order to illustrate the new contribution of our developments, we also decomposed the ship only with horizontal and vertical components, as it could be achieved with the super-elements developed so far. By so doing, we obtain the so-called "staircase" model depicted on Figure 12. If we apply the superelements method to this simple case, we obtain the curve $P(\delta)$ denoted by "staircase" model on Figure 
13. As it can be stated, this curve shows an important discrepancy with results given by LS-DYNA.

\subsection{Ship with strong side inclination}

For this second model of ship, we consider a much more inclined shell plating, with $\alpha=45^{\circ}$ (Fig. 14). We choose this particular configuration in order to show the importance of considering the side inclination when developing simplified analytical tools. Except this new value of $\alpha$, the ship is identical to the one presented in the previous section. In particular, the scantling is very similar to the one depicted on Figure 11.

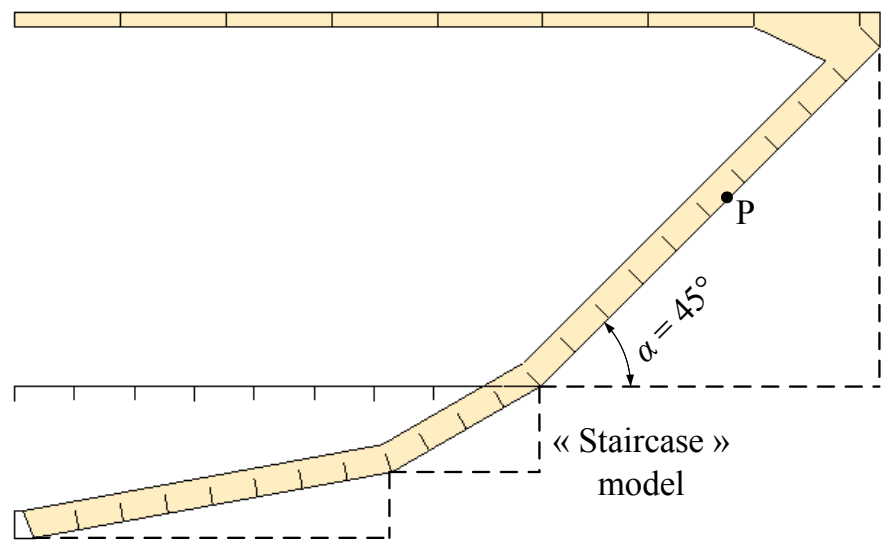

Figure 14. Section of ship model 2 and corresponding "staircase" model.

Comparisons between the numerical curve given by LS-DYNA and the analytical ones are shown on Figure 15. It is now clear that the "staircase" model is really inappropriate for modeling ships with strongly inclined plating. On the contrary, the "analytical" curve obtained with help of our new superelement appears to be much more suited.

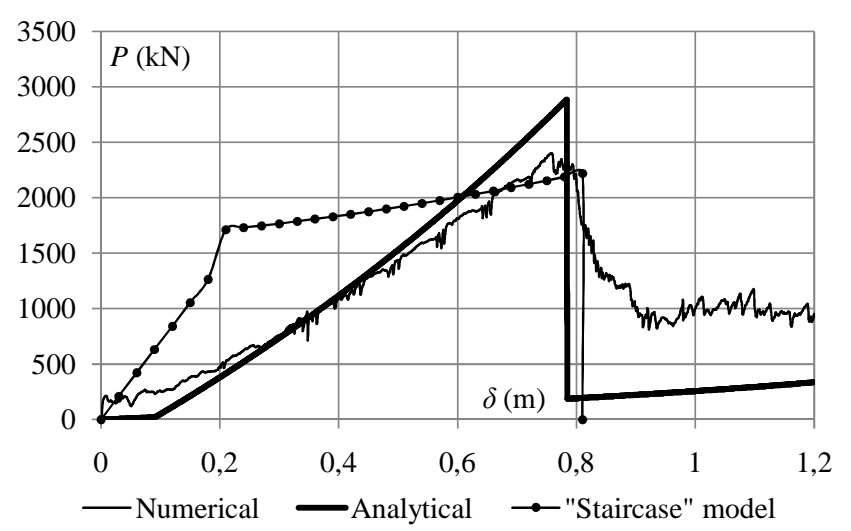

Figure 15. Section of ship model 2 and corresponding "staircase" model.

However, there is still a little discrepancy between the "analytical" and the "numerical" curves. As explained in section 4.1, this is still due to the coupling between the decks and the plating. In the present case, the divergence remains moderate because rup- ture occurs before having too large flexural displacements of the decks. But if we remove the failure condition in our models, we observe that the two curves exhibit a discrepancy increasing with the penetration $\delta$. For this reason, it seems to be important to perform additional research in order to account for this phenomenon.

\section{CONCLUSION}

In this paper, we present a new super-element developed to treat collisions on ships having an inclined shell plating. For such components, a relation between the global resisting force $P$ and the penetration of the striking vessel $\delta$ is derived.

The analytical developments are then verified by comparing the calculated crushing force with numerical results obtained by simulating collisions between a rigid bow and an oblique plate. We found a quite good agreement with the closed-form expression of $P(\delta)$ derived for the new super-element.

In order to check the coherence of our developments, collisions on full scale ship models are also considered. On one hand, we performed numerical simulations with LS-DYNA for a ship impacted by a rigid bow. On the other hand, we integrate our present developments in a global super-elements treatment of the ship. Comparisons between the results of the two approaches show a reasonable agreement.

However, simulations on entire models point out a lack in our calculations. When developing the law $P(\delta)$ for an inclined plate, we assumed that it is simply supported on its four edges, where displacements are constrained. On a real ship, this assumption has to be relaxed because the oblique plate is supported by bulkheads and decks. As these structures are not infinitely rigid, they may exhibit some bending deformation, especially when large tensile forces occur in the plating. As a consequence, the boundaries of the plate are likely to move, which implies a reduced internal dissipation in the superelement. In this sense, our calculations may not be conservative.

It is quite difficult to handle with this problem of coupling, because the super-elements method precisely assumed that each structural component is decoupled from the others. As a consequence, some additional research is still needed for a better integration of this phenomenon. Nevertheless, the developments exposed in this paper already allows for a first treatment of collisions on ships having inclined plating. In particular, results are much more confident than those obtained by modeling the vessel only with vertical and horizontal super-elements ("staircase" models). This however shows the real need of establishing new laws, as it has been done throughout this work. 


\section{APPENDIX}

In this appendix, we provide the mathematical expressions required to analytically evaluate the resistance of an impacted inclined plate. In this section, we will in fact detail equations (12) and (13).

The resisting force provided by the fibers parallel to $x$ axis is given by:

$$
P_{x}=\sigma_{0} t_{p} \sum_{i=1}^{12} P_{x, i}
$$

where the corresponding expressions for $1 \leq i \leq 6$ are as follow:

$$
\begin{aligned}
& P_{x, 1}=\frac{8 b_{2}^{2} \sin ^{2} \alpha}{3 x_{0} \cos \alpha}\left[1-\sqrt{f_{1}} \operatorname{arctanh}\left(\sqrt{\frac{1}{f_{1}}}\right)\right] \\
& P_{x, 2}=\frac{4 b_{2} \delta \sin ^{2} \alpha}{3 a_{1}}\left(1-\frac{1}{f_{1}}\right)^{-1} \\
& P_{x, 3}=\frac{4 R_{Z}}{3 R_{X}^{2}} \frac{b_{2}^{2} \sin ^{2} \alpha}{b_{2}-\delta \cos \alpha}\left[\sqrt{f_{2}} \arctan \left(\frac{a_{1}-x_{0}}{\sqrt{f_{2}}}\right)-f_{3}\right] \\
& P_{x, 4}=\frac{16 b_{1}^{2} \sin ^{2} \alpha}{3 x_{0} \cos \alpha}\left[1-\sqrt{f_{4}} \arctan \left(\sqrt{\frac{1}{f_{4}}}\right)\right] \\
& P_{x, 5}=\frac{4 b_{1} \delta \sin ^{2} \alpha}{3 a_{1}}\left(1-\frac{1}{f_{4}}\right)^{-1} \\
& P_{x, 6}=\frac{4 R_{Z}}{3 R_{X}^{2}} \frac{b_{1}^{2} \sin ^{2} \alpha}{b_{1}+\delta \cos \alpha}\left[\sqrt{f_{5}} \operatorname{arctanh}\left(\frac{a_{1}-x_{0}}{\sqrt{f_{2}}}\right)-f_{6}\right]
\end{aligned}
$$

For $7 \leq i \leq 12$, the formulae of $P_{x, i}$ are simply obtained by substituting $a_{1}$ by $a_{2}$ in the previous expressions of $P_{x, i}$ and $f_{i}$.

The resisting force provided by the fibers parallel to $y$ axis is given by:

$$
P_{y}=\sigma_{0} t_{p} \sum_{i=1}^{16} P_{y, i}
$$

where the corresponding expressions for $1 \leq i \leq 6$ are as follow:

$$
\begin{aligned}
& P_{y, 1}=\frac{3 a_{1} b_{2} \sin ^{2} \alpha}{2 \delta \cos ^{2} \alpha}\left[1-\sqrt{f_{1}} \operatorname{arctanh}\left(\sqrt{\frac{1}{f_{1}}}\right)\right] \\
& P_{y, 2}=\frac{x_{0} \sin ^{2} \alpha}{2 \cos \alpha}\left(1-\frac{1}{f_{1}}\right)^{-1} \\
& P_{y, 3}=\frac{b_{2}\left(b_{2}-2 \delta \cos \alpha\right) \sin ^{2} \alpha}{2\left(b_{2}-\delta \cos \alpha\right)^{2} \cos \alpha} \sqrt{f_{2}} \operatorname{arctanh}\left(f_{7}\right) \\
& P_{y, 4}=\frac{b_{2}^{2} \sin ^{2} \alpha}{2\left(b_{2}-\delta \cos \alpha\right)^{2} \cos \alpha}\left[\frac{a_{1}-x_{0}}{1+f_{7}^{2}}\right]
\end{aligned}
$$

$$
\begin{aligned}
& P_{y, 5}=\frac{3 a_{1} b_{1} \sin ^{2} \alpha}{2 \delta \cos ^{2} \alpha}\left[1-\sqrt{f_{4}} \arctan \left(\sqrt{\frac{1}{f_{4}}}\right)\right] \\
& P_{y, 6}=\frac{x_{0} \sin ^{2} \alpha}{2 \cos \alpha}\left(1+\frac{1}{f_{1}}\right)^{-1} \\
& P_{y, 7}=\frac{b_{1}\left(b_{1}+2 \delta \cos \alpha\right) \sin ^{2} \alpha}{2\left(b_{1}+\delta \cos \alpha\right)^{2} \cos \alpha} \sqrt{f_{5}} \operatorname{arctanh}\left(f_{8}\right) \\
& P_{y, 8}=\frac{-b_{1}^{2} \sin ^{2} \alpha}{2\left(b_{1}+\delta \cos \alpha\right)^{2} \cos \alpha}\left[\frac{a_{1}-x_{0}}{1-f_{8}^{2}}\right]
\end{aligned}
$$

with:

$$
\begin{aligned}
& f_{1}=\frac{a_{1} b_{2}}{x_{0} \delta \cos \alpha} \quad f_{4}=\frac{a_{1} b_{1}}{x_{0} \delta \cos \alpha} \\
& f_{2}=R_{X}^{2} \frac{b_{2}-\delta \cos \alpha}{R_{Z} \cos \alpha} \quad f_{5}=R_{X}^{2} \frac{b_{1}+\delta \cos \alpha}{R_{Z} \cos \alpha} \\
& f_{3}=\frac{a_{1}-x_{0}}{1+\left(a_{1}-x_{0}\right)^{2} f_{2}^{-1}} \quad f_{6}=\frac{a_{1}-x_{0}}{1+\left(a_{1}-x_{0}\right)^{2} f_{5}^{-1}} \\
& f_{7}=\frac{a_{1}-x_{0}}{\sqrt{f_{2}}} \quad f_{8}=\frac{a_{1}-x_{0}}{\sqrt{f_{5}}}
\end{aligned}
$$

For $9 \leq i \leq 16$, the formulae of $P_{y, i}$ are simply obtained by substituting $a_{1}$ by $a_{2}$ in the previous expressions of $P_{y, i}$ and $f_{i}$.

\section{REFERENCES}

Buldgen L., Le Sourne H., Besnard N. \& Rigo P. 2012, Extension of the super-elements method to the analysis of oblique collision between two ships, Marine Structures 29, 22-57.

Jones, N. 1997, Structural Impact, Cambridge University Press.

Le Sourne, H. 2001, Surface ship collisions: bibliography of fracture models used for fast dynamic finite elements simulations. Assessment of the threshold strain values to be used in a ship collision simulation, IRCN report 01/RT070.

Le Sourne, H. 2007, A ship collision analysis program based on super-element method coupled with large rotational ship movement analysis tool, Int. conf. on collision and grounding of ships ICCGS-2007, 131-138.

Lützen, M. 2001, Ship Collision Damage, PhD. diss., Technical University of Denmark.

Lützen, M., Simonsen, B.C. \& Pedersen, P.T. 2000, Rapid prediction of damage to struck and striking vessels in a collision event, Int. conf. on ship structure for the new millennium: supporting quality in shipbuilding.

Pedersen, P.T. \& Zhang S.M. 1998, On the mechanics in ship collisions, Marine Structures 11, 429-449.

Simonsen, B.C. 1997, Ship grounding on rock - I. Theory, Marine Structures 10, 519-562.

Zhang, S.M. 1999, The mechanics of ship collisions, PhD. diss., Technical University of Denmark. 